TRANSACTIONS OF THE

AMERICAN MATHEMATICAL SOCIETY

Volume 360, Number 8, August 2008, Pages 4125-4143

S 0002-9947(08)04368-7

Article electronically published on February 27, 2008

\title{
ORTHOGONAL POLYNOMIALS WITH A RESOLVENT-TYPE GENERATING FUNCTION
}

\author{
MICHAEL ANSHELEVICH
}

\begin{abstract}
The subject of this paper are polynomials in multiple non-commuting variables. For polynomials of this type orthogonal with respect to a state, we prove a Favard-type recursion relation. On the other hand, free Sheffer polynomials are a polynomial family in non-commuting variables with a resolvent-type generating function. Among such families, we describe the ones that are orthogonal. Their recursion relations have a more special form; the best way to describe them is in terms of the free cumulant generating function of the state of orthogonality, which turns out to satisfy a type of secondorder difference equation. If the difference equation is in fact first order, and the state is tracial, we show that the state is necessarily a rotation of a free product state. We also describe interesting examples of non-tracial infinitely divisible states with orthogonal free Sheffer polynomials.
\end{abstract}

\section{INTRODUCTION}

Let $\mathbf{x}=\left(x_{1}, \ldots, x_{n}\right), \mathbf{z}=\left(z_{1}, \ldots, z_{n}\right)$ be $n$-tuples of non-commuting indeterminates, such that the $x$-variables commute with the $z$-variables. Sometimes we will treat such $n$-tuples as vectors, in which case $\mathbf{x} \cdot \mathbf{z}$ denotes the scalar product.

Definition 1. Let

$$
F(\mathbf{z})=1+\text { higher-order terms }
$$

be a formal power series, and $\mathbf{V}$ be an $n$-tuple of formal power series,

$$
V_{i}(\mathbf{z})=z_{i}+\text { higher-order terms. }
$$

Expand

$$
F(\mathbf{z})(1-\mathbf{x} \cdot \mathbf{V}(\mathbf{z}))^{-1}
$$

into a power series in $z$. The coefficient of the monomial $z_{\vec{u}}$ is easily seen to be a monic polynomial $P_{\vec{u}}(\mathbf{x})$. We call $\left\{P_{\vec{u}}\right\}$ the (multivariate) free Sheffer polynomials.

The question to be investigated in this paper is: when are the free Sheffer polynomials orthogonal with respect to some state $\varphi$ ? We emphasize that $\left\{x_{1}, \ldots, x_{n}\right\}$ do not commute, and so one can talk about orthogonality of $\left\{P_{\vec{u}}\right\}$ only with respect to a positive functional on the algebra of non-commutative polynomials $\mathbb{R}\langle\mathbf{x}\rangle$, not with respect to a measure on $\mathbb{R}^{n}$.

The rest of the Introduction explains the motivation behind this question.

Received by the editors October 25, 2004 and, in revised form, June 7, 2006.

2000 Mathematics Subject Classification. Primary 05E35; Secondary 46L54, 33C47.

This work was supported in part by an NSF grant DMS-0400860.

(C)2008 American Mathematical Society Reverts to public domain 28 years from publication 
Let $\left\{P_{n}(x)\right\}$ be a monic polynomial family (in on variable) with a generating function of the form

$$
\sum_{n=0}^{\infty} P_{n}(x) z^{n}=\frac{1}{1-x U(z)+R(U(z))} .
$$

Here $U=z+$ higher-order terms and $R=z^{2}+$ higher-order terms are formal power series. The main theorem of Section 3 of [3] can be reformulated as follows.

Proposition 1. The polynomials with the generating function (1) are orthogonal if and only if the following two conditions hold:

(a) $U(z)=(R(z) / z)^{\langle-1\rangle}$, and

(b) $R(z) / z^{2}=1+b R(z) / z+c(R(z) / z)^{2}$.

Here $F^{\langle-1\rangle}$ denotes the inverse under composition.

Notice the similarity of this result to the following theorem, found in various forms by various people and going back to Meixner [15].

Proposition 2. Let $\left\{P_{n}(x)\right\}$ be a family of Sheffer polynomials, that is, a polynomial family with a generating function of the form

$$
\sum_{n=0}^{\infty} \frac{1}{n !} P_{n}(x) z^{n}=\exp (x U(z)-R(U(z))) .
$$

Here the conditions on $U$ and $R$ are the same as above. These polynomials are orthogonal if and only if the following two conditions hold:

(a) $U(z)=\left(R^{\prime}(z)\right)^{\langle-1\rangle}$, and

(b) $R^{\prime \prime}(z)=1+b R^{\prime}(z)+c\left(R^{\prime}(z)\right)^{2}$.

In fact, polynomials satisfying the conditions of Proposition 2 can be listed explicitly. They consist of polynomials orthogonal with respect to the Gaussian, Poisson, gamma, binomial, negative binomial, and continuous binomial (hyperbolic secant) distributions, all important in probability and statistics. It makes sense therefore to look at the polynomials with the generating function (11), which we call the free Sheffer polynomials, and in particular at the polynomials satisfying the conditions of Proposition 1 which we call the free Meixner polynomials. Here the adjective "free" refers to their relation to free probability [20]; see [3, 4] for more details. These polynomials can also be described explicitly; see Theorem 4 of [3]. They include Chebyshev polynomials of the 2nd kind, and other families whose orthogonality measure may include at most two atoms; they belong to the class investigated by Szegö and described on pages 26-28 of [5]. In particular, the semicircular, free Poisson (Marchenko-Pastur) and free binomial distributions are of this type. See Example 6 for more details.

The parallel between Propositions 1 and 2 can be explained by noticing that they are both particular cases of a more general theorem involving the generating function of a specific basic hypergeometric form; see [1] or Theorem 4.8 of [4. Proposition 1 is recovered for $q=0$, while Proposition 2 is recovered for $q=$ 1. The general family of orthogonal polynomials involved are the Al-Salam and Chihara polynomials; in particular, the (Rogers) continuous $q$-Hermite polynomials interpolate between the Hermite polynomials and the Chebyshev polynomials of the 2nd kind. 
Despite the similarity between single-variable Propositions 1 and2, the key point about Definition 1 is that it involves polynomials in non-commuting variables. In contrast, natural multivariate generalizations of Proposition 2 involve more familiar polynomials in commuting variables, orthogonal with respect to $n$-dimensional measures. They have been investigated by a number of people; see for example 11, 14, 8, 17, 9, 18. This analysis is usually performed in the context of natural exponential families. So this paper may be a precursor to "free statistics". For the moment, there are two other motivations for it. First, the hope is that these objects will turn out to play a role in free probability. Second, while there is some work on orthogonal polynomials in non-commuting variables [6], the field appears to be largely unexplored. In particular, while there are many interesting examples of multivariate orthogonal polynomials in commuting variables [10, there is a paucity of examples in the non-commutative case. The original motivation for this paper was to provide such examples. They come from free product states (Section 4), and from a certain exponentiation of a free semicircular system (Section 5).

\section{Preliminaries}

2.1. Polynomials. Let $\mathbb{R}\langle\mathbf{x}\rangle=\mathbb{R}\left\langle x_{1}, x_{2}, \ldots, x_{n}\right\rangle$ be all the polynomials with real

coefficients in $n$ non-commuting variables. Multi-indices are elements $\vec{u} \in\{1, \ldots, n\}^{k}$ for $k \geq 0$; for $|\vec{u}|=0$ we denote $\vec{u}$ by $\emptyset$. For two multi-indices $\vec{u}, \vec{v}$, denote by $(\vec{u}, \vec{v})$ their concatenation. For $\vec{u}$ with $|\vec{u}|=k$, denote

$$
(\vec{u})^{o p}=(u(k), \ldots, u(2), u(1)) .
$$

Define an involution on $\mathbb{R}\langle\mathbf{x}\rangle$ via an $\mathbb{R}$-linear extension of

$$
\left(x_{\vec{u}}\right)^{*}=x_{(\vec{u})^{o p}} .
$$

Here $x_{\vec{u}}$ is the monomial $x_{u(1)} \ldots x_{u(k)}$.

A monic polynomial family in $\mathbf{x}$ is a family $\left\{P_{\vec{u}}(\mathbf{x})\right\}$ indexed by all multi-indices

$$
\bigcup_{k=1}^{\infty}\left\{\vec{u} \in\{1, \ldots, n\}^{k}\right\}
$$

(with $P_{\emptyset}=1$ being understood) such that $\left\{P_{\vec{u}}(\mathbf{x})\right\}=x_{\vec{u}}+$ lower-order terms. Note that $P_{\vec{u}}^{*} \neq P_{(\vec{u})^{o p}}$ in general.

A polynomial family $\left\{P_{\vec{u}}\right\}$ is pseudo-orthogonal with respect to a functional $\varphi$ if

$$
\varphi\left[P_{\vec{u}}^{*} P_{\vec{v}}\right]=0
$$

whenever $|\vec{u}| \neq|\vec{v}|$ (including $\vec{u}=\emptyset$ ). The family is orthogonal if this is the case whenever $\vec{u} \neq \vec{v}$. Note that an orthogonal polynomial family $\left\{P_{\vec{u}}\right\}$ determines its unital functional of orthogonality $\varphi$ via $\varphi[1]=1, \varphi\left[P_{\vec{u}}\right]=0$, so such a functional, if it exists, is unique.

Most theorems about formal power series remain valid for non-commuting variables. In particular, a series $F(\mathbf{z})=1+$ higher-order terms has a unique inverse with respect to multiplication, always denoted by $F^{-1}$. Also, an $n$-tuple of series $\mathbf{U}$ with $U_{i}(\mathbf{z})=z_{i}+$ higher-order terms has a unique inverse with respect to composition, always denoted by $\mathbf{U}^{\langle-1\rangle}$. 
2.2. Linear functionals and free cumulants. Let $\varphi$ be a unital real linear functional on $\mathbb{R}\langle\mathbf{x}\rangle$. It can be thought of as a moment functional of the variables $\left\{x_{1}, \ldots, x_{n}\right\}$. Here their joint moments are

$$
M\left[x_{\vec{u}}\right]=\varphi\left[x_{\vec{u}}\right]=\varphi\left[x_{u(1)} \ldots x_{u(k)}\right] .
$$

Denote by

$$
M(\mathbf{z})=\sum_{k=1}^{\infty} \sum_{|\vec{u}|=k} M\left[x_{\vec{u}}\right] z_{\vec{u}}=\varphi\left[(1-\mathbf{x} \cdot \mathbf{z})^{-1}\right]-1
$$

the ordinary moment generating function of $\varphi$. Here, and in the sequel,

$$
\mathbf{z}=\left(z_{1}, z_{2}, \ldots, z_{n}\right)
$$

are formal non-commuting indeterminates, which commute with the $\left\{x_{i}\right\}$. Note that $M(\mathbf{z})$ completely determines $\varphi$.

The free cumulant functional $R$ corresponding to $\varphi$ is usually defined using the lattice of non-crossing partitions: $R[1]=0$ and for $|\vec{u}|=k$,

$$
R\left[x_{\vec{u}}\right]=M\left[x_{\vec{u}}\right]-\sum_{\substack{\pi \in N C(k) \\ \pi \neq 1}} \prod_{B \in \pi} R\left[\prod_{i \in B} x_{u(i)}\right],
$$

which expresses $R\left[x_{\vec{u}}\right]$ in terms of the joint moments and sums of products of lower-order free cumulants. From these, we can form the free cumulant generating function via

$$
R(\mathbf{z})=\sum_{k=1}^{\infty} \sum_{|\vec{u}|=k} R\left[x_{\vec{u}}\right] z_{\vec{u}} .
$$

However, in this paper we will not use non-crossing partitions. So for the rest of the paper, we take as the definition of free cumulants the following implicit functional relation; see Section 13 of [16] or Proposition 3.1 of [4]:

$$
R\left(w_{1}(1+M(\mathbf{w})), \ldots, w_{n}(1+M(\mathbf{w}))\right)=M(\mathbf{w}) .
$$

To simplify notation, we will assume throughout the paper that the $\left\{x_{i}\right\}$ are centered and have unit covariance,

$$
R\left[x_{i}\right]=\varphi\left[x_{i}\right]=0
$$

and

$$
R\left[x_{i} x_{j}\right]=\varphi\left[x_{i} x_{j}\right]-\varphi\left[x_{i}\right] \varphi\left[x_{j}\right]=\delta_{i j} .
$$

The results can be modified for more general (in particular, degenerate) covariance, but the formulas become more complicated.

A state on $\mathbb{R}\langle\mathbf{x}\rangle$ is a linear functional that is unital (that is, $\varphi[1]=1$ ) and positive, that is, for any polynomial $A(\mathbf{x})$,

$$
\varphi\left[A(\mathbf{x})^{*} A(\mathbf{x})\right] \geq 0 .
$$

Such a functional cannot always be extended from $\mathbb{R}\langle\mathbf{x}\rangle$ to a state on some $C^{*}$ algebra. This is already true in the commutative case: a positive functional on $\mathbb{R}\left[x_{1}, x_{2}\right]$ need not come from a positive measure on $\mathbb{R}^{2}$. The issue is whether the moment problem is solvable; for an example of a non-commutative result, see [13.

A state is faithful if $\varphi\left[A(\mathbf{x})^{*} A(\mathbf{x})\right]=0$ only for $A(\mathbf{x})=0$. We will only consider faithful states in this paper; but see Remark 3 . 
For unital linear functionals

$$
\left\{\varphi_{i} \text { on } \mathbb{R}\left[x_{i}\right]\right\}_{i=1}^{n},
$$

their free product functional $\varphi$ on $\mathbb{R}\left\langle x_{1}, x_{2}, \ldots, x_{n}\right\rangle$ is defined by the requirement that

$$
R_{\varphi}\left[x_{\vec{u}}\right]=0
$$

unless all $u(j)$ are equal (that is, mixed free cumulants are zero), and

$$
R_{\varphi}\left[x_{i}^{k}\right]=R_{\varphi_{i}}\left[x_{i}^{k}\right] .
$$

$\varphi$ is a state if $\varphi_{i}$ 's are. Conversely, if $\varphi$ happens to be of this form, we say that $\left\{x_{i}\right\}$ are freely independent with respect to it. See 20] or [19] for a lot more about this, and in particular for an explanation of the terminology. If a similar definition is given for the algebra of commutative polynomials in terms of the usual cumulants, one obtains exactly product states, corresponding to product measures, and the notion of independence.

Example 1. If $\varphi_{1}$ is a state on $\mathbb{R}\left[x_{1}\right], \varphi_{2}$ is a state on $\mathbb{R}\left[x_{2}\right]$, and $\varphi$ is their free product state on $\mathbb{R}\left\langle x_{1}, x_{2}\right\rangle$, then

$$
\begin{aligned}
\varphi\left[x_{1} x_{2}+x_{1} x_{2} x_{1}+x_{1} x_{2} x_{1} x_{2}\right] & =\varphi_{1}\left[x_{1}\right] \varphi_{2}\left[x_{2}\right]+\varphi_{1}\left[x_{1}^{2}\right] \varphi_{2}\left[x_{2}\right] \\
+ & \left(\varphi_{1}\left[x_{1}\right]^{2} \varphi_{2}\left[x_{2}^{2}\right]+\varphi_{1}\left[x_{1}^{2}\right] \varphi_{2}\left[x_{2}\right]^{2}-\varphi_{1}\left[x_{1}\right]^{2} \varphi_{2}\left[x_{2}\right]^{2}\right) .
\end{aligned}
$$

2.3. Operators. Define the left partial derivative with respect to $z_{i}, D_{i}$ by

$$
\begin{gathered}
D_{i}(1)=0, \\
D_{i} z_{j}=\delta_{i j}, \\
D_{i}\left(z_{j} z_{\vec{u}}\right)=\delta_{i j} z_{\vec{u}} .
\end{gathered}
$$

Denote by $\mathbf{D}=\left(D_{1}, D_{2}, \ldots, D_{n}\right)$ the left gradient.

Given a monic polynomials family $\left\{P_{\vec{u}}\right\}$, define the right partial lowering operator with respect $x_{i}, L_{i}$, by

$$
\begin{gathered}
L_{i}(1)=0, \\
L_{i} P_{j}(\mathbf{x})=\delta_{i j}, \\
L_{i} P_{(\vec{u}, j)}(\mathbf{x})=\delta_{i j} P_{\vec{u}}(\mathbf{x}) .
\end{gathered}
$$

\section{Free Meixner families}

Proposition 3. Monic polynomials are orthogonal with respect to some faithful state if and only if they satisfy a recursion

$$
x_{i} P_{\vec{u}}=P_{(i, \vec{u})}+\sum_{|\vec{w}|=|\vec{u}|} B_{i, \vec{w}, \vec{u}} P_{\vec{w}}+\sum_{|\vec{v}|=|\vec{u}|-1} C_{i, \vec{v}, \vec{u}} P_{\vec{v}}
$$

with

(a) $C_{i, \vec{s}, \vec{u}}=0$ unless $\vec{u}=(i, \vec{s})$, and $C_{i, \vec{s},(i, \vec{s})}>0$,

(b) denoting $\vec{s}_{j}=(s(j), \ldots, s(k))$,

$$
B_{i, \vec{s}, \vec{u}} \prod_{j=1}^{k} C_{s(j), \vec{s}_{j+1}, \vec{s}_{j}}=B_{i, \vec{u}, \vec{s}} \prod_{j=1}^{k} C_{u(j), \vec{u}_{j+1}, \vec{u}_{j}} .
$$


Proof. First assume that the polynomials are orthogonal with respect to some faithful state $\varphi$. Denote

$$
\langle S(\mathbf{x}), T(\mathbf{x})\rangle=\varphi\left[S(\mathbf{x})^{*} T(\mathbf{x})\right] .
$$

Since the polynomials are monic, for any fixed $\vec{u}, i$,

$$
x_{i} P_{\vec{u}}=P_{(i, \vec{u})}+\sum_{|\vec{v}| \leq|\vec{u}|} \alpha_{i, \vec{v}, \vec{u}} P_{\vec{v}}
$$

for some coefficients $\alpha_{i, \vec{v}, \vec{u}}$. Also,

$$
\left\langle P_{\vec{v}}, x_{i} P_{\vec{u}}\right\rangle=\varphi\left[P_{\vec{v}}^{*}\left(x_{i} P_{\vec{u}}\right)\right]=\varphi\left[\left(x_{i} P_{\vec{v}}\right)^{*} P_{\vec{u}}\right]=\left\langle x_{i} P_{\vec{v}}, P_{\vec{u}}\right\rangle=0
$$

for $|\vec{v}| \leq|\vec{u}|-2$. It follows that the polynomials satisfy a recursion of the type (4).

In that case, for general $\vec{u}$ and $\vec{s}$

$$
\begin{aligned}
\left\langle x_{i} P_{\vec{u}}, P_{\vec{s}}\right\rangle & =\left\langle P_{(i, \vec{u})}, P_{\vec{s}}\right\rangle+\sum_{|\vec{w}|=|\vec{u}|} B_{i, \vec{w}, \vec{u}}\left\langle P_{\vec{w}}, P_{\vec{s}}\right\rangle+\sum_{|\vec{v}|=|\vec{u}|-1} C_{i, \vec{v}, \vec{u}}\left\langle P_{\vec{v}}, P_{\vec{s}}\right\rangle \\
=\left\langle P_{\vec{u}}, x_{i} P_{\vec{s}}\right\rangle & =\left\langle P_{\vec{u}}, P_{(i, \vec{s})}\right\rangle+\sum_{|\vec{w}|=|\vec{s}|} B_{i, \vec{w}, \vec{s}}\left\langle P_{\vec{u}}, P_{\vec{w}}\right\rangle+\sum_{|\vec{v}|=|\vec{s}|-1} C_{i, \vec{v}, \vec{s}}\left\langle P_{\vec{u}}, P_{\vec{v}}\right\rangle .
\end{aligned}
$$

Pseudo-orthogonality implies that for $|\vec{s}|=|\vec{u}|-1$

$$
\sum_{|\vec{v}|=|\vec{u}|-1} C_{i, \vec{v}, \vec{u}}\left\langle P_{\vec{v}}, P_{\vec{s}}\right\rangle=\left\langle P_{\vec{u}}, P_{(i, \vec{s})}\right\rangle
$$

and for $|\vec{s}|=|\vec{u}|$

$$
\sum_{|\vec{w}|=|\vec{u}|} B_{i, \vec{w}, \vec{u}}\left\langle P_{\vec{w}}, P_{\vec{s}}\right\rangle=\sum_{|\vec{w}|=|\vec{s}|} B_{i, \vec{w}, \vec{s}}\left\langle P_{\vec{u}}, P_{\vec{w}}\right\rangle
$$

(the case $|\vec{s}|=|\vec{u}|+1$ is redundant). Using the orthogonality assumption,

$$
C_{i, \vec{s}, \vec{u}} V_{\vec{s} \vec{s}}=\delta_{\vec{u},(i, \vec{s})} V_{\vec{u} \vec{u}}
$$

and

$$
B_{i, \vec{s}, \vec{u}} V_{\vec{s} \vec{s}}=B_{i, \vec{u}, \vec{s}} V_{\vec{u} \vec{u}}
$$

where

$$
V_{\vec{u} \vec{u}}=\left\langle P_{\vec{u}}, P_{\vec{u}}\right\rangle
$$

It follows that

$$
\begin{gathered}
V_{\vec{u} \vec{u}}=\prod_{j=1}^{k} C_{u(j), \vec{u}_{j+1}, \vec{u}_{j}}, \\
C_{i, \vec{s}, \vec{u}} \prod_{j=1}^{k-1} C_{s(j), \vec{s}_{j+1}, \vec{s}_{j}}=\delta_{\vec{u},(i, \vec{s})} \prod_{j=1}^{k} C_{u(j), \vec{u}_{j+1}, \vec{u}_{j}}
\end{gathered}
$$

and

$$
B_{i, \vec{s}, \vec{u}} \prod_{j=1}^{k} C_{s(j), \vec{s}_{j+1}, \vec{s}_{j}}=B_{i, \vec{u}, \vec{s}} \prod_{j=1}^{k} C_{u(j), \vec{u}_{j+1}, \vec{u}_{j}} .
$$

Equation (8) is condition (b). Equation (7) is equivalent to requiring that $C_{i, \vec{s}, \vec{u}}=0$ unless $\vec{u}=(i, \vec{s})$, and faithfulness of $\varphi$ implies that $C_{i, \vec{s},(i, \vec{s})}>0$, which together form condition (a). 
Conversely, assume that the polynomials satisfy the recursion (4) with the conditions of the proposition. On $\mathbb{R}\langle\mathbf{x}\rangle$, define the functional $\varphi$ by requiring that the induced inner product

$$
\langle S(\mathbf{x}), T(\mathbf{x})\rangle=\varphi\left[S(\mathbf{x})^{*} T(\mathbf{x})\right]
$$

satisfies

$$
\left\langle P_{\vec{u}}, P_{\vec{v}}\right\rangle=\delta_{\vec{u} \vec{v}} V_{\vec{u} \vec{u}}= \begin{cases}0 & \text { if } \vec{u} \neq \vec{v}, \\ V_{\vec{u} \vec{u}} & \text { if } \vec{u}=\vec{v},\end{cases}
$$

where $V_{\vec{u} \vec{u}}$ is now defined via equation (6), and extending linearly. So for $S(\mathbf{x})=$ $\sigma_{\emptyset}+\sum_{\vec{u}} \sigma_{\vec{u}} P_{\vec{u}}(\mathbf{x}), T(\mathbf{x})=\tau_{\emptyset}+\sum_{\vec{u}} \tau_{\vec{u}} P_{\vec{u}}(\mathbf{x})$,

$$
\langle S(\mathbf{x}), T(\mathbf{x})\rangle=\sigma_{\emptyset} \tau_{\emptyset}+\sum_{\vec{u}} \sigma_{\vec{u}} \tau_{\vec{u}} V_{\vec{u} \vec{u}} .
$$

If this functional is well-defined, the given polynomials are orthogonal with respect to it. Also, since $V_{\vec{u} \vec{u}}$ are positive, the functional will be positive and faithful.

To show that this definition is consistent, we need to show that if

$$
S(\mathbf{x}) T(\mathbf{x})=S^{\prime}(\mathbf{x}) T^{\prime}(\mathbf{x})
$$

then

$$
\left\langle S^{*}, T\right\rangle=\left\langle\left(S^{\prime}\right)^{*}, T^{\prime}\right\rangle
$$

For $\mathbb{R}\langle\mathbf{x}\rangle$, the fundamental theorem of algebra no longer holds, but these polynomials still form a Unique Factorization Domain. Thus the equality (9) reduces to the situation $(Q S) T=Q(S T)$. By linearity, we may assume that $S$ is a monomial. But in that case, by iteration we may assume that $S=x_{i}$. Finally, by linearity again we may assume that $Q^{*}, T$ are basis polynomials. Thus we only need to satisfy the following condition:

$$
\left\langle x_{i} P_{\vec{u}}, P_{\vec{s}}\right\rangle=\left\langle P_{\vec{u}}, x_{i} P_{\vec{s}}\right\rangle
$$

which, using the recursion relation, is equivalent to equation (5). The arguments from the first half of the proof imply that this equality holds provided that conditions (a), (b) are satisfied.

Remark 2. It follows from the proof of the preceding proposition that any pseudoorthogonal polynomials satisfy a recursion of type (4).

Remark 3. If the appropriate part of condition (a) of the proposition is replaced by the condition $C_{i, \vec{s},(i, \vec{s})} \geq 0$, it follows that the corresponding polynomials are still orthogonal with respect to a state that need not be faithful. The converse characterization is an interesting question that is not treated in this paper.

Lemma 4. Let $\left\{P_{\vec{u}}\right\}$ be a family of free Sheffer polynomials as in Definition 1, with

$$
H(\mathbf{x}, \mathbf{z})=1+\sum_{\vec{u}} P_{\vec{u}}(\mathbf{x}) z_{\vec{u}}=F(\mathbf{z})(1-\mathbf{x} \cdot \mathbf{V}(\mathbf{z}))^{-1}
$$

Assume more particularly that $F(\mathbf{z})=1-\sum_{i=1}^{n} z_{i}^{2}+$ higher-order terms. Define the functional $\varphi$ on $\mathbb{R}\left\langle x_{1}, x_{2}, \ldots, x_{n}\right\rangle$ by $\varphi[1]=1, \varphi\left[P_{\vec{u}}\right]=0$ for $|\vec{u}| \geq 1$. Then in fact,

$$
H(\mathbf{x}, \mathbf{z})=(1-\mathbf{x} \cdot \mathbf{U}(\mathbf{z})+R(\mathbf{U}(\mathbf{z})))^{-1}
$$


where $R(\mathbf{z})$ is the free cumulant generating function of $\varphi$, and $U_{i}(\mathbf{z})=V_{i}(\mathbf{z}) F^{-1}(\mathbf{z})$. We say that $\left\{P_{\vec{u}}\right\}$ is the free Sheffer family associated to the functional $\varphi$ and the functions U. Note that if a free Sheffer family is orthogonal, it is orthogonal with respect to the functional $\varphi$ to which it is associated.

Proof. By definition of $\varphi$ and $H, \varphi[H(\mathbf{x}, \mathbf{z})]=1$. Then

$$
1=F(\mathbf{z}) \varphi\left[(1-\mathbf{x} \cdot \mathbf{U}(\mathbf{z}) F(\mathbf{z}))^{-1}\right]=F(\mathbf{z})(1+M(\mathbf{U}(\mathbf{z}) F(\mathbf{z}))) .
$$

Since the $n$-tuple of power series $\mathbf{U}$ is invertible under composition, we may write

$$
F(\mathbf{z})=(1+K(\mathbf{U}(\mathbf{z})))^{-1}
$$

for some power series $K$. Then

$$
1+K(\mathbf{U})=1+M\left(\mathbf{U}(1+K(\mathbf{U}))^{-1}\right) .
$$

Therefore from equation (3),

$$
\begin{aligned}
& R\left(U_{1}(1+K(\mathbf{U}))^{-1}\left(1+M\left(\mathbf{U}(1+K(\mathbf{U}))^{-1}\right)\right)\right. \\
& \left.\quad \ldots, U_{n}(1+K(\mathbf{U}))^{-1}\left(1+M\left(\mathbf{U}(1+K(\mathbf{U}))^{-1}\right)\right)\right)=K(\mathbf{U}) .
\end{aligned}
$$

However, this expression also equals

$$
R\left(U_{1}(1+K(\mathbf{U}))^{-1}(1+K(\mathbf{U})), \ldots, U_{n}(1+K(\mathbf{U}))^{-1}(1+K(\mathbf{U}))\right)=R(\mathbf{U}) .
$$

Thus $F(\mathbf{z})=(1+R(\mathbf{U}(\mathbf{z})))^{-1}$ and

$$
F(\mathbf{z})(1-\mathbf{x} \cdot \mathbf{V}(\mathbf{z}))^{-1}=(1-\mathbf{x} \cdot \mathbf{U}(\mathbf{z})+R(\mathbf{U}(\mathbf{z})))^{-1} .
$$

Proposition 5 (4, Theorem 3.21]). Suppose that a family of free Sheffer polynomials is pseudo-orthogonal. Then for $R, \mathbf{U}$ as in Lemma 4 ,

$$
\left(D_{i} R\right)(\mathbf{U}(\mathbf{z}))=z_{i} .
$$

Remark 4. Both $\mathbf{D} R$ and $\mathbf{U}$ are $n$-tuples of non-commutative power series invertible under composition. So

(a) Given $R$, the preceding proposition completely determines $\mathbf{U}$, and vice versa. From now on, we will always assume this relationship between $R$ and $\mathbf{U}$.

(b) Since the inverse under composition is unique, also

$$
U_{i}((\mathbf{D} R)(\mathbf{z}))=z_{i} .
$$

Definition 2. A state $\varphi$ on $\mathbb{R}\langle\mathbf{x}\rangle$ is called a free Meixner state if, for $R$ its free cumulant generating function and $\mathbf{U}$ determined by the preceding remark, the free Sheffer polynomials with the generating function

$$
(1-\mathbf{x} \cdot \mathbf{U}(\mathbf{z})+R(\mathbf{U}(\mathbf{z})))^{-1}
$$

are orthogonal. 
Theorem 6. Suppose that a family of free Sheffer polynomials is pseudo-orthogonal. Then

(a) The power series $\mathbf{U}$ satisfy the relation

$$
z_{j}=U_{j}+\sum_{i, t} B_{i j}^{t} U_{i} z_{t}+\sum_{i, s, t} C_{i j}^{s t} U_{i} z_{s} z_{t} .
$$

In other words, denoting by $A$ the matrix

$$
I+\sum_{t} B^{t} z_{t}+\sum_{s, t} C^{s t} z_{s} z_{t}
$$

$\mathbf{U}=\mathbf{z} A^{-1}$

(b) The polynomials satisfy the recursion

$$
\begin{gathered}
x_{i} P_{s}=P_{(i, s)}+\sum_{j} B_{i j}^{s} P_{j}+\delta_{i s}, \\
x_{i} P_{(s, t, \vec{u})}=P_{(i, s, t, \vec{u})}+\sum_{j} B_{i j}^{s} P_{(j, t, \vec{u})}+\sum_{j}\left(\delta_{i s} \delta_{j t}+C_{i j}^{s t}\right) P_{(j, \vec{u})} .
\end{gathered}
$$

(c) The free cumulant generating function satisfies

$$
D_{i} D_{j} R(\mathbf{z})=\delta_{i j}+\sum_{t} B_{i j}^{t} D_{t} R(\mathbf{z})+\sum_{s, t} C_{i j}^{s t} D_{s} R(\mathbf{z}) D_{t} R(\mathbf{z}) .
$$

Proof. By definition of the function $H$ in Lemma 4 ,

$$
L_{j} H(\mathbf{x}, \mathbf{z})=H(\mathbf{x}, \mathbf{z}) z_{j} .
$$

Also from that lemma,

$$
(1+R(\mathbf{U}(\mathbf{z}))) H=(\mathbf{x} \cdot \mathbf{U}(\mathbf{z})) H+1 .
$$

Applying $L_{j}$ to this expression, we get

$L_{j}((x \cdot \mathbf{U}(\mathbf{z})) H)=L_{j}((1+R(\mathbf{U}(\mathbf{z}))) H)=(1+R(\mathbf{U}(\mathbf{z}))) H z_{j}=(\mathbf{x} \cdot \mathbf{U}(\mathbf{z})) H z_{j}+z_{j}$.

Expanding $H$ in powers of $z$, we get

$$
L_{j}\left(\sum_{i} x_{i} U_{i}(z)\left(1+\sum_{\vec{u}} P_{\vec{u}} z_{\vec{u}}\right)\right)=\sum_{i} x_{i} U_{i}(z)\left(1+\sum_{\vec{u}} P_{\vec{u}} z_{\vec{u}}\right) z_{j}+z_{j},
$$

and so

$$
U_{j}+\sum_{\vec{u}, i} L_{j}\left(x_{i} P_{\vec{u}}\right) U_{i}(z) z_{\vec{u}}=z_{j}+\sum_{i} x_{i} U_{i}(z) z_{j}+\sum_{\vec{u}, i} x_{i} P_{\vec{u}} U_{i}(z) z_{\vec{u}} z_{j},
$$

where we used the fact that $L_{j}\left(x_{i}\right)=L_{j}\left(P_{i}\right)=\delta_{i j}$.

Since $U_{i}=z_{i}+$ higher-order terms,

$$
z_{j}=U_{j}+\sum_{i, \vec{u}} a_{i, j, \vec{u}} U_{i}(z) z_{\vec{u}}
$$

for some coefficients $\left\{a_{i, j, \vec{u}}\right\}$. Using equation (10),

$$
D_{j} R=z_{j}+\sum_{i, \vec{u}} a_{i, j, \vec{u}} z_{i}(\mathbf{D} R)_{\vec{u}}
$$


where $(\mathbf{D} R)_{\vec{u}}=\left(D_{u(1)} R\right)\left(D_{u(2)} R\right) \ldots\left(D_{u(k)} R\right)$. Therefore

$$
D_{i} D_{j} R=\delta_{i j}+\sum_{\vec{u}} a_{i, j, \vec{u}}(\mathbf{D} R)_{\vec{u}} .
$$

Combining equations (11) and (12),

$$
\begin{aligned}
U_{j}+\sum_{i, \vec{u}} L_{j}\left(x_{i} P_{\vec{u}}\right) U_{i}(z) z_{\vec{u}}=U_{j} & +\sum_{i, \vec{u}} a_{i, j, \vec{u}} U_{i}(z) z_{\vec{u}} \\
& +\sum_{i} x_{i} U_{i}(z) z_{j}+\sum_{i, \vec{u}} x_{i} P_{\vec{u}} U_{i}(z) z_{\vec{u}} z_{j} .
\end{aligned}
$$

Equating coefficients of $U_{i} z_{\vec{u}}$,

$$
L_{j}\left(x_{i} P_{\vec{u}}\right)=a_{i, j, \vec{u}}+\delta_{u(k), j} x_{i} P_{(u(1), \ldots, u(k-1))} .
$$

Since the polynomials are pseudo-orthogonal, they satisfy a recursion relation (4). So

$$
L_{j}\left(x_{i} P_{\vec{u}}\right)=\delta_{u(k), j} P_{(i, u(1), \ldots, u(k-1))}+\sum_{|\vec{w}|=|\vec{u}|-1} B_{i,(\vec{w}, j), \vec{u}} P_{\vec{w}}+\sum_{|\vec{v}|=|\vec{u}|-2} C_{i,(\vec{v}, j), \vec{u}} P_{\vec{v}} .
$$

Combining the two preceding equations with equation (4) for $x_{i} P_{(u(1), \ldots, u(k-1))}$, we get

$$
\begin{aligned}
& a_{i, j, \vec{u}}+\delta_{u(k), j}\left(P_{(i, u(1), \ldots, u(k-1))}\right.+\sum_{|\vec{w}|=|\vec{u}|-1} B_{i, \vec{w},(u(1), \ldots, u(k-1))} P_{\vec{w}} \\
&\left.+\sum_{|\vec{v}|=|\vec{u}|-2} C_{i, \vec{v},(u(1), \ldots, u(k-1))} P_{\vec{v}}\right) \\
&=\delta_{u(k), j} P_{(i, u(1), \ldots, u(k-1))}+\sum_{|\vec{w}|=|\vec{u}|-1} B_{i,(\vec{w}, j), \vec{u}} P_{\vec{w}}+\sum_{|\vec{v}|=|\vec{u}|-2} C_{i,(\vec{v}, j), \vec{u}} P_{\vec{v}} .
\end{aligned}
$$

Equating coefficients,

$$
\begin{aligned}
a_{i, j, \vec{u}}= & \sum_{|\vec{w}|=|\vec{u}|-1}\left(B_{i,(\vec{w}, j), \vec{u}}-\delta_{u(k), j} B_{i, \vec{w},(u(1), \ldots, u(k-1))}\right) P_{\vec{w}} \\
& +\sum_{|\vec{v}|=|\vec{u}|-2}\left(C_{i,(\vec{v}, j), \vec{u}}-\delta_{u(k), j} C_{i, \vec{v},(u(1), \ldots, u(k-1))}\right) P_{\vec{v}} .
\end{aligned}
$$

In particular, for $\vec{u}=t$ this says

$$
a_{i, j, t}=B_{i, j, t}-\delta_{j t} B_{i, \emptyset, \emptyset}
$$

and for $\vec{u}=(s, t)$ this says

$$
a_{i, j,(s, t)}=\sum_{w}\left(B_{i,(w, j),(s, t)}-\delta_{j t} B_{i, w, s}\right) P_{w}+\left(C_{i, j,(s, t)}-\delta_{j t} C_{i, \emptyset, s}\right) .
$$

Therefore

$$
\begin{gathered}
B_{i,(\vec{w}, j), \vec{u}}=\delta_{u(k), j} B_{i, \vec{w},(u(1), \ldots, u(k-1))} ; \quad B_{i, j, t}=\delta_{j t} B_{i, \emptyset, \emptyset}+a_{i, j, t}, \\
C_{i,(\vec{v}, j), \vec{u}}=\delta_{u(k), j} C_{i, \vec{v},(u(1), \ldots, u(k-1))} ; \quad C_{i, j,(s, t)}=\delta_{j t} C_{i, \emptyset, s}+a_{i, j,(s, t)} .
\end{gathered}
$$


So

$$
\begin{aligned}
B_{i,(j, \vec{u}),(t, \vec{u})} & =\delta_{j t} B_{i, \emptyset, \emptyset}+a_{i, j, t}, \\
C_{i,(j, \vec{u}),(s, t, \vec{u})} & =\delta_{j t} C_{i, \emptyset, s}+a_{i, j,(s, t)}
\end{aligned}
$$

and zero otherwise.

$$
\begin{gathered}
x_{i}=P_{i}+B_{i, \emptyset, \emptyset}, \\
x_{i} P_{t}=P_{(i, t)}+\sum_{s} B_{i, s, t} P_{s}+C_{i, \emptyset, t} .
\end{gathered}
$$

So $B_{i, \emptyset, \emptyset}=R\left[x_{i}\right]=0, C_{i, \emptyset, t}=R\left[x_{i} x_{t}\right]=\delta_{i t}$. Also, $a_{i, j, \vec{u}}=0$ for $|\vec{u}|>2$. Denote $B_{i j}^{t}=a_{i, j, t}, C_{i j}^{s t}=a_{i, j,(s, t)}$. Part (b) follows. For parts (a) and (c), use equations (12), (13), respectively.

Corollary 7. Let $\varphi$ be a state, $R$ its free cumulant generating function, $\mathbf{U}$ the corresponding power series determined by Proposition 5 , and $\left\{P_{\vec{u}}\right\}$ the corresponding free Sheffer polynomials. $\varphi$ is a faithful free Meixner state if and only if the following equivalent conditions hold:

$$
z_{j}=U_{j}+\sum_{i, t} B_{i j}^{t} U_{i} z_{t}+\sum_{i} C_{i j} U_{i} z_{i} z_{j}
$$

or

$$
D_{i} D_{j} R=\delta_{i j}+\sum_{t} B_{i j}^{t} D_{t} R+C_{i j} D_{i} R D_{j} R
$$

or

$$
\begin{gathered}
x_{i} P_{t}=P_{(i, t)}+\sum_{j} B_{i j}^{t} P_{j}+\delta_{i t}, \\
x_{i} P_{(t, \vec{u})}=P_{(i, t, \vec{u})}+\sum_{j} B_{i j}^{t} P_{(j, \vec{u})}+\delta_{i t}\left(1+C_{i, u(1)}\right) P_{\vec{u}} .
\end{gathered}
$$

In all cases, the coefficients have to satisfy

(a) $C_{i j}>-1$.

(b) $B_{i j}^{t}=B_{i t}^{j}$.

(c) For each $j, t$, either $B_{i j}^{t}=0$ for all $i$, or $C_{j u}=C_{t u}$ for all $u$.

Proof. If the free Sheffer polynomials are orthogonal with respect to the state $\varphi$, then in particular $\varphi\left[P_{\vec{u}}\right]=0$, so by Lemma $4, \varphi$ is exactly the state with the free cumulant generating function $R$.

Combine Proposition 3 with Theorem [6. It follows that $C_{s t}^{i j}=\delta_{i s} \delta_{j t} C_{i j}$ and

$$
C_{i, \vec{u},(i, \vec{u})}=1+C_{i, u(1)}
$$

and zero otherwise, so condition (a) follows from Proposition 3(a). Also,

$$
B_{i,(j, \vec{u}),(t, \vec{u})} V_{(j, \vec{u}),(j, \vec{u})}=B_{i,(t, \vec{u}),(j, \vec{u})} V_{(t, \vec{u}),(t, \vec{u})},
$$

so

For $|\vec{u}|=0$, this says

$$
B_{i j}^{t} V_{(j, \vec{u}),(j, \vec{u})}=B_{i t}^{j} V_{(t, \vec{u}),(t, \vec{u})} .
$$

$$
B_{i j}^{t}=B_{i t}^{j},
$$

implying condition (b). For longer $\vec{u}$, this says

$$
B_{i j}^{t} C_{j, \vec{u},(j, \vec{u})}=B_{i t}^{j} C_{t, \vec{u},(t, \vec{u})},
$$


SO

implying condition (c).

$$
B_{i j}^{t}\left(1+C_{j u}\right)=B_{i t}^{j}\left(1+C_{t u}\right),
$$

Conversely, suppose that for the state $\varphi$ and the corresponding free Sheffer polynomials $\left\{P_{\vec{u}}\right\}$, the recursion (15) with conditions (a)-(c) holds. Then by Proposition 3 the polynomials are orthogonal, necessarily with respect to $\varphi$, and $\varphi$ is faithful. The equivalence of the conditions for $R, \mathbf{U}$, and the polynomials in the corollary follows from Theorem 6 .

\section{First-order, tracial Case}

Throughout this section, we will assume that the state $\varphi$ is tracial, that is, for any $S, T$,

$$
\varphi[S(\mathbf{x}) T(\mathbf{x})]=\varphi[T(\mathbf{x}) S(\mathbf{x})] .
$$

This produces two simplifications. First, for any $\vec{u}, i$,

$$
R\left[x_{\vec{u}} x_{i}\right]=R\left[x_{i} x_{\vec{u}}\right] .
$$

This is not apparent from the definition of $R$ via equation (3), but follows easily from the definition using non-crossing partitions.

Second, any pseudo-orthogonal polynomials can be orthogonalized (with real coefficients).

Remark 5. Starting with an arbitrary monic polynomial family, by using the GramSchmidt procedure it can be transformed into a pseudo-orthogonal family; note that this family is still monic. Given an ordering of the monomials of the same degree, the procedure can be applied further to produce an orthogonal family. However, this will necessarily destroy the monic condition. Therefore, the condition that monic orthogonal polynomials exist is rather strong, and does not hold for all tracial states.

Lemma 8. Let $B, C$ be as in Theorem [6. $B_{i j}^{t}$ is invariant under cyclic permutations of $(j, i, t)$, and $\sum_{t} B_{i j}^{t} B_{c t}^{d}+C_{i j}^{c d}$ is invariant under cyclic permutations of $(j, i, c, d)$.

Proof. By assumption,

$$
D_{i} D_{j} R=\delta_{i j}+\sum_{t} B_{i j}^{t} D_{t} R+\sum_{s, t} C_{i j}^{s t} D_{s} R D_{t} R
$$

and also

$$
R=\sum_{i} z_{i}^{2}+\sum R\left[x_{a} x_{b} x_{c}\right] z_{a} z_{b} z_{c}+\sum R\left[x_{a} x_{b} x_{c} x_{d}\right] z_{a} z_{b} z_{c} z_{d}+\ldots
$$

Then

$$
D_{j} R=z_{j}+\sum R\left[x_{j} x_{b} x_{c}\right] z_{b} z_{c}+\sum R\left[x_{j} x_{b} x_{c} x_{d}\right] z_{b} z_{c} z_{d}+\ldots
$$

and so

$$
\begin{aligned}
D_{i} D_{j} R & =\delta_{i j}+\sum R\left[x_{j} x_{i} x_{c}\right] z_{c}+\sum R\left[x_{j} x_{i} x_{c} x_{d}\right] z_{c} z_{d}+\ldots \\
& =\delta_{i j}+\sum B_{i j}^{t} z_{t}+\sum B_{i j}^{t} R\left[x_{t} x_{b} x_{c}\right] z_{b} z_{c}+\sum C_{i j}^{c d} z_{c} z_{d}+\ldots
\end{aligned}
$$

It follows that

$$
R\left[x_{j} x_{i} x_{t}\right]=B_{i j}^{t}
$$


and

$$
R\left[x_{j} x_{i} x_{c} x_{d}\right]=\sum_{t} B_{i j}^{t} R\left[x_{t} x_{c} x_{d}\right]+C_{i j}^{c d}=\sum_{t} B_{i j}^{t} B_{c t}^{d}+C_{i j}^{c d} .
$$

So the result follows from cyclic symmetry (16).

Lemma 9. Let $O$ be an orthogonal transformation on $\mathbb{R}^{n}$. Perform changes of variables $\mathbf{x}=O \mathbf{y}, \mathbf{w}=O^{-1} \mathbf{z}$. Then

$$
\begin{gathered}
R_{\mathbf{x}}(\mathbf{z})=R_{\mathbf{y}}(\mathbf{w}), \\
\mathbf{D} R_{\mathbf{x}}(\mathbf{z})=O \mathbf{D} R_{\mathbf{y}}(\mathbf{w}), \\
Q R_{\mathbf{x}}(\mathbf{z})=O\left(Q R_{\mathbf{y}}(\mathbf{w})\right) O^{-1},
\end{gathered}
$$

where $Q_{i j} R=D_{i} D_{j} R$.

$$
\left(1-\mathbf{x} \cdot \mathbf{U}(\mathbf{z})+R_{\mathbf{x}}(\mathbf{U}(\mathbf{z}))\right)^{-1}=\left(1-\mathbf{y} \cdot \mathbf{V}(\mathbf{w})+R_{\mathbf{y}}(\mathbf{V}(\mathbf{w}))\right)^{-1}
$$

for $\mathbf{V}(\mathbf{w})=O^{-1} \mathbf{U}(O \mathbf{w})$. The induced functional on $\mathbb{R}\langle\mathbf{y}\rangle$ is tracial if $\varphi$ is tracial. The polynomials with the generating function (17) are orthogonal for all such changes of variable $O$ if and only if, in addition to the conditions of Corollary 7 , $C_{i j} \equiv c$.

Proof.

$$
R_{\mathbf{x}}(\mathbf{z})=\sum_{\vec{u}} R\left[x_{\vec{u}}\right] z_{\vec{u}} .
$$

So by linearity of $R$, for $x_{i}=\sum_{j} O_{i j} y_{j}$,

$$
\begin{aligned}
R_{\mathbf{x}}(\mathbf{z})=\sum_{\vec{u}} \sum_{|\vec{v}|=|\vec{u}|} \prod_{i=1}^{k} O_{u(i) v(i)} R\left[y_{\vec{v}}\right] z_{\vec{u}} & =\sum_{\vec{v}} R\left[y_{\vec{v}}\right] \sum_{|\vec{v}|=|\vec{u}|} \prod_{i=1}^{k} O_{u(i) v(i)} z_{\vec{u}} \\
& =\sum_{\vec{v}} R\left[y_{\vec{v}}\right] w_{\vec{v}}=R_{\mathbf{y}}(\mathbf{w}),
\end{aligned}
$$

where $w_{j}=\sum_{i} O_{i j} z_{i}$. Also,

$$
\begin{aligned}
D_{i} R_{\mathbf{x}}(\mathbf{z}) & =\sum_{\vec{v}} R\left[y_{\vec{v}}\right] \sum_{|\vec{v}|=|\vec{u}|+1} O_{i v(1)} \prod_{j=2}^{k} O_{u(j) v(j)} z_{\vec{u}} \\
& =\sum_{\vec{v}, s} R\left[y_{s} y_{\vec{v}}\right] O_{i s} w_{\vec{v}}=\sum_{s} O_{i s} D_{s} R_{\mathbf{y}}(\mathbf{w}) .
\end{aligned}
$$

Similarly,

$$
D_{i} D_{j} R_{\mathbf{x}}(\mathbf{z})=\sum_{s, t} O_{i s} O_{j t} D_{s} D_{t} R_{\mathbf{y}}(\mathbf{w}) .
$$

Equation (17) follows. The tracial property is clear.

If

$$
D_{i} D_{j} R_{\mathbf{x}}(\mathbf{z})=\delta_{i j}+\sum_{t} B_{i j}^{t} D_{t} R_{\mathbf{x}}(\mathbf{z})+C_{i j} D_{i} R_{\mathbf{x}}(\mathbf{z}) D_{j} R_{\mathbf{x}}(\mathbf{z}),
$$

then

$$
\begin{aligned}
D_{i} D_{j} R_{\mathbf{y}}(\mathbf{w})=\delta_{i j} & +\sum B_{\alpha \beta}^{t} O_{\alpha i} O_{\beta j} O_{t s} D_{s} R_{\mathbf{y}}(\mathbf{w}) \\
& +\sum C_{s t} O_{s i} O_{t j} O_{s u} O_{t v} D_{u} R_{\mathbf{y}}(\mathbf{w}) D_{v} R_{\mathbf{y}}(\mathbf{w}) .
\end{aligned}
$$


For orthogonality of the induced free Sheffer polynomials in $\mathbf{y}$, we check the conditions of Corollary 7 , By Lemma 8 ,

$$
B_{\alpha \beta}^{t} O_{\alpha i} O_{\beta j} O_{t s}=B_{\alpha t}^{\beta} O_{\alpha i} O_{\beta j} O_{t s}=B_{\alpha \beta}^{t} O_{\alpha i} O_{t j} O_{\beta s},
$$

so this expression is symmetric in $j, s$. On the other hand, we also need

$$
\sum_{s, t} C_{s t} O_{s i} O_{t j} O_{s u} O_{t v}=\delta_{i u} \delta_{j v} E_{i j} .
$$

Taking the sum of these expressions with respect to $\sum_{i, j} O_{a i} O_{b j}$, we get

$$
C_{a b} O_{a u} O_{b v}=E_{u v} O_{a u} O_{b v} .
$$

It follows that for all $a, b, u, v, C_{a b}=E_{u v}$, hence $C_{a b} \equiv c$. Finally, for constant $C$ the last condition of the corollary is trivially true.

Corollary 10. Let $B, C$ be as in Corollary 7 . Then $B_{i j}^{t}$ is symmetric under all permutations of $(i, j, t)$, and $C_{i j}$ is symmetric in its arguments. If $C \equiv 0$, all the matrices $\left\{B^{t}\right\}$ commute.

Proof. The symmetry of $B_{i j}^{t}$ comes by combining the cyclic symmetry from Lemma 8 with the transposition symmetry from Corollary 7 . Also from that lemma,

$$
R\left[x_{j} x_{i} x_{i} x_{j}\right]=\sum_{t} B_{i j}^{t} B_{i t}^{j}+C_{i j},
$$

while

$$
R\left[x_{i} x_{j} x_{j} x_{i}\right]=\sum_{t} B_{j i}^{t} B_{j t}^{i}+C_{j i} .
$$

It follows that $C_{j i}=C_{i j}$. Using the cyclic symmetry from the lemma again and setting $C \equiv 0$,

$$
\sum_{t} B_{i j}^{t} B_{c t}^{d}=\sum_{t} B_{c i}^{t} B_{d t}^{j}
$$

So

$$
\left(B^{j} B^{c}\right)_{i d}=\sum_{t} B_{i t}^{j} B_{t d}^{c}=\sum_{t} B_{i t}^{c} B_{t d}^{j}=\left(B^{c} B^{j}\right)_{i d} .
$$

Example 6 (Product states). Let $\varphi^{b, c}$ be a one-dimensional free Meixner state, that is, the state on $\mathbb{R}[x]$ whose free cumulant generating function satisfies the equation in Proposition 1. The solution of this equation is

$$
R(z)=z \frac{z^{-1}-b-\sqrt{\left(z^{-1}-b\right)^{2}-4 c}}{2 c} .
$$

Note that the free cumulant generating function $R$ differs from a more familiar $R$-transform by a factor of $z \cdot \varphi^{b, c}$ itself can be identified with the measure

$$
\frac{1}{2 \pi} \frac{\sqrt{4(1+c)-(x-b)^{2}}}{1+b x+c x^{2}} d x+\text { zero, one, or two atoms; }
$$

see Theorem 4 of 3 , for a more detailed description, with different normalizations. Here $b \in \mathbb{R}$, and $c \geq-1$ (for $c=-1$, the measure is purely atomic, so the corresponding state is not faithful). In particular, the free Gamma case corresponds to $b^{2}=4 c$, the free Poisson case to $c=0$, and the free Gaussian (semicircular) case to $b=c=0$. See also [7] for related results. 
Let $\varphi$ be the free product state of $\left\{\varphi^{b_{i}, c_{i}}, i=1, \ldots, n\right\}$. The free cumulant generating function of $\varphi$ is simply

$$
R(\mathbf{z})=\sum_{i=1}^{n} R_{i}\left(z_{i}\right)
$$

where $R_{i}$ is the free cumulant generating function of $\varphi^{b_{i}, c_{i}}$, satisfying

$$
R_{i}\left(z_{i}\right) / z_{i}^{2}=1+b_{i} R_{i}\left(z_{i}\right) / z_{i}+c_{i}\left(R_{i}\left(z_{i}\right) / z_{i}\right)^{2} .
$$

Let $U_{i}(\mathbf{z})=\left(R_{i}\left(z_{i}\right) / z_{i}\right)^{\langle-1\rangle}$. Then the free Sheffer polynomials corresponding to $(R, \mathbf{U})$ are orthogonal. Indeed, these polynomials satisfy the recursion

$$
\begin{gathered}
y_{i} P_{(j, \vec{u})}=P_{(i, j, \vec{u})}, \\
y_{i} P_{(i, j, \vec{u})}=P_{(i, i, j, \vec{u})}+b_{i} P_{(i, j, \vec{u})}+P_{(j, \vec{u})}, \\
y_{i} P_{(i, i, \vec{u})}=P_{(i, i, i, \vec{u})}+b_{i} P_{(i, i, \vec{u})}+\left(1+c_{i}\right) P_{(i, \vec{u})},
\end{gathered}
$$

for $i \neq j$. So

$$
B_{i,(\alpha, \beta, \vec{w}),(s, t, \vec{u})}=\delta_{\vec{w}, \vec{u}} \delta_{s i} \delta_{\alpha s} \delta_{\beta t} b_{i}
$$

and

$$
C_{i,(\alpha, \vec{w}),(s, t, \vec{u})}=\delta_{\vec{w}, \vec{u}} \delta_{s i} \delta_{\alpha t}\left(1+\delta_{t i} c_{i}\right) .
$$

The conditions of Proposition 3 are satisfied, so the polynomials are orthogonal.

Explicitly, these polynomials are free products. Denote by $\left\{P_{k}^{b, c}\right\}$ the onevariable free Meixner polynomials from Proposition 1 . Decompose a multi-index $\vec{u}$ so that

$$
x_{\vec{u}}=x_{v(1)}^{i(1)} x_{v(2)}^{i(2)} \ldots x_{v(k)}^{i(k)},
$$

where the consecutive indices $v(j) \neq v(j+1)$, although non-consecutive indices may coincide. Then

$$
P_{\vec{u}}(\mathbf{x})=\prod_{j=1}^{k} P_{i(j)}^{b_{v(j)}, c_{v(j)}}\left(x_{v(j)}\right) .
$$

Thus free products of one-dimensional free Meixner states are free Meixner. The following proposition provides a partial converse.

Proposition 11. Suppose that $\varphi$ is a tracial free Meixner state with

$$
D_{i} D_{j} R_{\varphi}=\delta_{i j}+\sum_{t} B_{i j}^{t} D_{t} R_{\varphi}
$$

Then up to a rotation, $\varphi$ is a free product state of semicircular and free Poisson distributions.

Proof. It follows from Corollary 10 that the matrices $\left\{B^{r}\right\}$ are all symmetric and mutually commuting. So we can find an orthogonal transformation $O$ such that $\left(O^{-1} B^{r} O\right)_{i j}=\delta_{i j} b_{i}^{r}$ for all $r$. Performing the change of variable in Lemma 9, we get

So

$$
O\left(Q R_{\mathbf{y}}(\mathbf{w})\right) O^{-1}=I+B \cdot O \mathbf{D} R_{\mathbf{y}}(\mathbf{w}) .
$$

$$
Q R_{\mathbf{y}}(\mathbf{w})=I+O^{-1}\left(B \cdot O \mathbf{D} R_{\mathbf{y}}(\mathbf{w})\right) O .
$$

Note that $B_{i j}^{k}=\sum O_{i s} b_{s}^{k} O_{j s}$ is also equal to $B_{i k}^{j}=\sum O_{i s} b_{s}^{j} O_{k s}$. Then

$$
\left(O^{-1}(B \cdot O \mathbf{w}) O\right)_{\alpha \beta}=\sum_{k, l} \delta_{\alpha \beta} b_{\alpha}^{k} O_{k l} w_{l} .
$$


On the other hand, it is also equal to

$$
\sum_{i, j, s, k, l} O_{i \alpha} O_{i s} b_{s}^{j} O_{k s} O_{j \beta} O_{k l} w_{l}=\sum_{j} b_{\alpha}^{j} O_{j \beta} w_{\alpha} .
$$

As a result, $\sum_{k} \delta_{\alpha \beta} b_{\alpha}^{k} O_{k l}=\sum_{j} \delta_{\alpha l} b_{\alpha}^{j} O_{j \beta}$ and

$$
\left(O^{-1}(B \cdot O \mathbf{w}) O\right)_{\alpha \beta}=\delta_{\alpha \beta}\left(\sum b_{\alpha}^{k} O_{k \alpha}\right) w_{\alpha} .
$$

Denote $b_{\alpha}=\sum_{k} b_{\alpha}^{k} O_{k \alpha}$. Then

$$
\left.D_{i} D_{j} R_{\mathbf{y}}(\mathbf{w})=\delta_{i j}+\delta_{i j} b_{i} D_{i} R_{\mathbf{y}}(\mathbf{w})=\delta_{i j}\left(1+b_{i} D_{i} R_{\mathbf{y}}(\mathbf{w})\right)\right) .
$$

Therefore

$$
R_{\mathbf{y}}(\mathbf{w})=\sum_{i=1}^{n} R_{y_{i}}\left(w_{i}\right)
$$

so all the mixed cumulants are zero and the components are freely independent. Moreover, each $R_{i}$ satisfies the equation

$$
R_{i} / w_{i}^{2}=1+b_{i} R_{i} / w_{i} .
$$

This is exactly the equation in Proposition 1 for the free Poisson case, or for the semicircular case if $b_{i}=0$.

\section{A Freely infinitely Divisible EXAmPle}

Definition 3. A state $\varphi$ is freely infinitely divisible if for all $t>0$, the functional $\varphi^{t}$ with the free cumulant generating function

$$
R_{\varphi^{t}}(\mathbf{z})=t R_{\varphi}(\mathbf{z})
$$

is also positive definite.

Remark 7. One-dimensional free Meixner states $\varphi^{b, c}$ of Proposition 1 and Example 6 are freely infinitely divisible for $c \geq 0$, and are not freely infinitely divisible for $-1 \leq c<0$. In fact, in this case $\varphi^{t}$ is a state only for $t \geq-c$.

Thus all the states of Proposition 11 are freely infinitely divisible, but some more general free product states of Example 6 are not. In this section, we construct an example of a freely infinitely divisible free Meixner state that is not a free product state.

Definition 4. A functional $\psi$ on $\mathbb{R}\langle\mathbf{x}\rangle$ is conditionally positive definite if it is positive definite on polynomials of degree at least 2 .

Lemma 12. $\varphi$ is freely infinitely divisible if and only if its free cumulant functional is conditionally positive definite.

Proof. $R_{\varphi}\left[x_{\vec{u}}\right]=\left.\frac{d}{d t}\right|_{t=0} M_{\varphi^{t}}\left[x_{\vec{u}}\right]$. So if each $\varphi^{t}$ is conditionally positive definite, so is $R_{\varphi}$. For the converse, starting with a conditionally positive linear functional, one constructs symmetric operators with the joint distribution $\varphi$. See [12] or Section 4 of $[2$.

The following lemma is reminiscent of the Kolmogorov representation for infinitely divisible measures with finite variance. 
Lemma 13. Let $\left\{\varphi_{i}, i=1, \ldots, n\right\}$ be positive definite functionals on $\mathbb{R}\langle\mathbf{x}\rangle$. Define the functional $\psi$ on $\mathbb{R}\langle\mathbf{x}\rangle$ as follows:

$$
\begin{gathered}
\psi[1]=\psi\left[x_{i}\right]=0, \\
\psi\left[x_{i} x_{j}\right]=\delta_{i j}, \\
\psi\left[x_{i} P(\mathbf{x}) x_{j}\right]=\delta_{i j} \varphi_{i}[P(\mathbf{x})] .
\end{gathered}
$$

Then $\psi$ is conditionally positive definite.

Proof. For such $\psi$,

$$
\begin{aligned}
\psi\left[\left(\sum_{i} P_{i}(\mathbf{x}) x_{i}\right)^{*}\left(\sum_{j} P_{j}(\mathbf{x}) x_{j}\right)\right] & =\psi\left[\left(\sum_{i} x_{i} P_{i}(\mathbf{x})^{*}\right)\left(\sum_{j} P_{j}(\mathbf{x}) x_{j}\right)\right] \\
& =\psi\left[\sum_{i} x_{i} P_{i}(\mathbf{x})^{*} P_{i}(\mathbf{x}) x_{i}\right] \\
& =\sum_{i} \varphi_{i}\left[P_{i}(\mathbf{x})^{*} P_{i}(\mathbf{x})\right] \geq 0
\end{aligned}
$$

so $\psi$ is conditionally positive definite.

We will denote $\psi$ as above by $\exp \left(\varphi_{1} \oplus \ldots \oplus \varphi_{n}\right)$.

The following result was already used in the proof of Theorem 3.21 of [4]; here we formulate it as a lemma. Considering how different the relation (3) is from the logarithmic relation between moments and the usual cumulants, this result is surprisingly similar to the identity $(\log f)^{\prime}=f^{\prime} / f$.

Lemma 14. For $z_{i}=w_{i}(1+M(\mathbf{w}))$, we have

$$
(1+M(\mathbf{w})) D_{z_{i}} R(\mathbf{z})=D_{w_{i}} M(\mathbf{w}) .
$$

Proof. The result follows immediately from the relation (3).

Proposition 15. Let $\psi$ be the distribution of a free semicircular system with means $b_{i}$ and variances $c_{i}$. In other words, $\psi$ is the state with the free cumulants

$$
R_{\psi}\left[x_{i}\right]=b_{i}, \quad R_{\psi}\left[x_{i}^{2}\right]=c_{i},
$$

and all the other free cumulants are zero. Define the state $\varphi$ by $R_{\varphi}=\exp \left(\psi^{\oplus n}\right)$. Then $\varphi$ is a free Meixner state.

Proof. By definition,

$$
R_{\psi}(\mathbf{z})=\sum_{i}\left(b_{i} z_{i}+c_{i} z_{i}^{2}\right) .
$$

So

$$
D_{i} R_{\psi}(\mathbf{z})=b_{i}+c_{i} z_{i}
$$

Using the change of variables $z_{k}=w_{k}\left(1+M_{\psi}(\mathbf{w})\right)$ and Lemma 14, we get

$$
\left(1+M_{\psi}(\mathbf{w})\right)^{-1} D_{i} M_{\psi}(\mathbf{w})=b_{i}+c_{i} w_{i}\left(1+M_{\psi}(\mathbf{w})\right),
$$

and so

$$
D_{i} M_{\psi}(\mathbf{w})=b_{i}\left(1+M_{\psi}(\mathbf{w})\right)+c_{i}\left(1+M_{\psi}(\mathbf{w})\right) w_{i}\left(1+M_{\psi}(\mathbf{w})\right) .
$$

The combination of Lemmas 12 and 13 shows that $\varphi$ is a well-defined freely infinitely divisible state. Its free cumulant generating function is

$$
R_{\varphi}(\mathbf{w})=\sum_{j} w_{j}\left(1+M_{\psi}(\mathbf{w})\right) w_{j}
$$


Then

$$
D_{j} R_{\varphi}(\mathbf{w})=\left(1+M_{\psi}(\mathbf{w})\right) w_{j}
$$

and

$$
\begin{aligned}
D_{i} D_{j} R_{\varphi}(\mathbf{w}) & =\delta_{i j}+D_{i} M_{\psi}(\mathbf{w}) w_{j} \\
& =\delta_{i j}+b_{i}\left(1+M_{\psi}(\mathbf{w})\right) w_{j}+c_{i}\left(1+M_{\psi}(\mathbf{w})\right) w_{i}\left(1+M_{\psi}(\mathbf{w})\right) w_{j} \\
& =\delta_{i j}+b_{i} D_{j} R_{\varphi}(\mathbf{w})+c_{i} D_{i} R_{\varphi}(\mathbf{w}) D_{j} R_{\varphi}(\mathbf{w}) .
\end{aligned}
$$

Thus $B_{i j}^{t}=\delta_{j t} b_{i}$, the conditions of Corollary 7 are satisfied, and the free Sheffer polynomials corresponding to $\varphi$ are orthogonal.

Note that unless all $b_{i}=c_{i}=0, \varphi$ is not a tracial state.

\section{REFERENCES}

[1] Al-Salam, W. A. and Chinara, T. S. (1987). $q$-Pollaczek polynomials and a conjecture of Andrews and Askey. SIAM J. Math. Anal. 18 228-242. MR871834(88a:33019)

[2] Anshelevich, M. (2001). Partition-dependent stochastic measures and $q$-deformed cumulants. Doc. Math. 6 343-384 (electronic). MR1871667 (2004k:46107)

[3] Anshelevich, M. (2003). Free martingale polynomials. J. Funct. Anal. 201 228-261. MR:1986160 (2004f:46079)

[4] Anshelevich, M. (2004). Appell polynomials and their relatives. Int. Math. Res. Not. n.65, 3469-3531. MR2101359 (2005k:33012)

[5] Askey, R. and Wilson, J. (1985). Some basic hypergeometric orthogonal polynomials that generalize Jacobi polynomials. Mem. Amer. Math. Soc. 54 iv+55. MR783216 (87a:05023)

[6] Banks, T., Constantinescu, T. and Johnson, J. L. (2005). Relations on non-commutative variables and associated orthogonal polynomials. In Operator theory, systems theory and scattering theory: multidimensional generalizations, vol. 157 of Oper. Theory Adv. Appl. Birkhäuser, Basel, 61-90. MR2129643 (2006d:47012)

[7] Bożejko, M. and Bryc, W. (2006). On a class of free Lévy laws related to a regression problem. J. Funct. Anal. 236 59-77. MR2227129 (2007a:46071)

[8] CASAlis, M. (1991). Les familles exponentielles à variance quadratique homogène sont des lois de Wishart sur un cône symétrique. C. R. Acad. Sci. Paris Sér. I Math. 312 537-540. MR1099688 (91m:60029)

[9] Casalis, M. (1996). The $2 d+4$ simple quadratic natural exponential families on $\mathbf{R}^{d}$. Ann. Statist. 24 1828-1854. MR1416663 (97h:60011)

[10] DunkL, C. F. and XU, Y. (2001). Orthogonal polynomials of several variables, vol. 81 of Encyclopedia of Mathematics and its Applications. Cambridge University Press, Cambridge. MR:1827871 (2002m:33001)

[11] Feinsilver, P. (1986). Some classes of orthogonal polynomials associated with martingales. Proc. Amer. Math. Soc. 98 298-302. MR854037(88d:60136)

[12] Glockner, P., Schürmann, M. and Speicher, R. (1992). Realization of free white noises. Arch. Math. (Basel) 58 407-416. MR.1152632 (93e:46075)

[13] Hadwin, D. (2001). A noncommutative moment problem. Proc. Amer. Math. Soc. 129 17851791 (electronic). MR.1814111(2003a:46101)

[14] Letac, G. (1989). Le problème de la classification des familles exponentielles naturelles de $\mathbf{R}^{d}$ ayant une fonction variance quadratique. In Probability measures on groups, IX (Oberwolfach, 1988), vol. 1379 of Lecture Notes in Math. Springer, Berlin, 192-216. MR1020532(92a:60041)

[15] Meixner, J. (1934). Orthogonale polynomsysteme mit einer besonderen gestalt der erzeugenden funktion. J. London Math. Soc. 9 6-13.

[16] NicA, A. (1999). $R$-transforms in free probability. Lecture notes for an IHP course (unpublished).

[17] Pommeret, D. (1996). Orthogonal polynomials and natural exponential families. Test 5 77-111. MR 1410457 (97j:62016)

[18] Pommeret, D. (2000). Orthogonality of the Sheffer system associated to a Levy process. J. Statist. Plann. Inference 86 1-10. MR.1763177(2003b:60063) 
[19] Speicher, R. (1997). Free probability theory and non-crossing partitions. Sém. Lothar. Combin. 39 Art. B39c, 38 pp. (electronic). MR1490288 (98m:46081)

[20] Voiculescu, D. (2000). Lectures on free probability theory. In Lectures on probability theory and statistics (Saint-Flour, 1998), vol. 1738 of Lecture Notes in Math. Springer, Berlin, 279-349. MR.1775641 (2001g:46121)

Department of Mathematics, University of California, Riverside, California 925210135

Current address: Department of Mathematics, Texas A\&M University, College Station, Texas 77843-3368

E-mail address: manshel@math.tamu.edu 